\title{
Indicadores bibliométricos sobre o ensino por competências na Educação em Engenharia
}

DOI: 10.37702/2175-957X.COBENGE.2021.3747

Mariana Salgado Lopes - lopes_ms@outlook.com

Universidade Federal de São Carlos

Rua Abrahão João 435

13562-150 - São Carlos - SP

Humberto Dias - humbertodaf@outlook.com

Universidade Federal de São Carlos

Rua Edmar Neves, 550

39800-350 - Teófilo Otoni - MG

Daniel Rodrigo Leiva - daniel.leiva@ufscar.br

Universidade Federal de São Carlos

Rodovia Washington Luis, km 235 ---

13565-905 - São Carlos - SP

Resumo: Buscando atender as demandas do mercado de trabalho e modernizar os cursos de graduação em engenharia, foram publicadas, em 2019, as novas Diretrizes Curriculares Nacionais do Curso de Graduação baseadas em uma nova concepção de ensino focada na construção de competências. Ademais, está em vigor, o Programa Brasil-Estados Unidos de Modernização da Educação Superior na Graduação, que visa, entre outros objetivos, fomentar a construção de competências como criatividade, inovação e empreendedorismo. Desta forma, torna-se urgente um maior entendimento sobre a aplicação do ensino por competências na educação em engenharia. Com o intuito de trazer luz a este tema e determinar seu impacto científico, este trabalho se propôs a elaborar indicadores bibliométricos, a partir dos registros indexados à base de dados Web of Science, no período de 2001 à 2020. Foram analisados resultados sobre o número de publicações por ano, mostrando um crescimento do número de publicações ao longo do tempo. Além disso, quanto à produtividade por país, instituição e autor, os EUA se mostrou predominante neste campo de pesquisa, sendo o país responsável pela grande maioria dos registros no período analisado. Foi constatada uma significativa representatividade feminina na área, além da detecção de tendências de pesquisas que aliam o ensino por competências ao uso 


\section{COBENGE

de metodologias ativas, sendo tema para os próximos trabalhos uma investigação mais detalhada sobre quais as metodologias ativas mais abordadas e quais os melhores métodos para avaliar sua efetividade.

Palavras-chave: Ensino por competências. Educação em engenharia. Bibliometria. Indicadores bibliométricos. 


\section{INDICADORES BIBLIOMÉTRICOS SOBRE O ENSINO POR COMPETÊNCIAS NA EDUCAÇÃO EM ENGENHARIA}

\section{INTRODUÇÃO}

Diversos debates vêm ocorrendo ao redor do mundo, nos últimos anos, no campo de Educação em Engenharia, acerca da necessidade de modernizar o conteúdo dos cursos de graduação para atender às necessidades do mercado de trabalho. Há uma grande demanda do setor produtivo por profissionais que possuam, não só uma base técnica sólida, mas uma formação ampla e integrada, que lhes permita construir as competências necessárias para dialogar com outras áreas do conhecimento, absorver novas tecnologias, liderar equipes multidisciplinares, propor soluções viáveis e inovadoras para problemas complexos, atuar de forma ética e sustentável, empreender e se aprimorar continuamente.

Buscando atender a esses anseios, foram publicadas no Brasil, em 2019, as novas Diretrizes Curriculares Nacionais do Curso de Graduação em Engenharia (DCNs), construídas através de uma parceria inédita entre o governo, a comunidade acadêmica e o setor privado. Entre os objetivos das novas DCNs, pode-se destacar a melhoria do ensino em Engenharia no país, a partir da substituição de uma concepção de formação focada na transmissão de conteúdo, por uma formação voltada à construção de competências; na qual, o aluno passa a ser um agente fundamental no processo de aprendizagem, realizando atividades práticas e ativas, elaboradas em articulação com o setor produtivo, de forma a simular o cotidiano da profissão. Desta forma, é possível, tanto atender as demandas futuras por mais e melhores engenheiros, quanto estimular o engajamento dos alunos, diminuindo as elevadas taxas de evasão dos cursos de engenharia (BRASIL, 2019; CNI et al., 2020).

Ademais, está em funcionamento, desde 2019, o Programa Brasil-Estados Unidos de Modernização da Educação Superior na Graduação (PMG - EUA), com a participação inicial de oito cursos de engenharia de instituições de ensino superior (IES) brasileiras e duração prevista de 8 anos. O programa têm como objetivos, formar redes de colaboração internacional; oferecer oficinas de treinamento com especialistas para criar modelos de currículos, metodologias de ensino e gestão de curso aplicáveis à realidade das instituições nacionais; fomentar a construção de competências como criatividade, inovação e empreendedorismo e aproximar a universidade da comunidade externa, criando parcerias com empresas, para que os alunos tenham a oportunidade de praticar a resolução de problemas reais, utilizando os conhecimentos adquiridos na graduação (CAPES; COMISSÃO FULBRIGHT, 2018).

Considerando que o processo de transição para a formação baseada na construção de competências já está em vigor no país, torna-se urgente um maior entendimento sobre a aplicação do ensino por competências na Educação em Engenharia.

Uma das formas de acessar o conhecimento sobre determinado tema de pesquisa e determinar seu alcance e impacto é através do uso de indicadores de ciência e tecnologia, uma vez que, estes oferecem de forma simplificada e de fácil visualização, informações sobre a produtividade de instituições e autores, acompanhamento do número de publicações ao longo do tempo, identificação de tendências de pesquisa e contextualização da produtividade dos países em âmbito global, entre outros aspectos. Assim sendo, a bibliometria constitui uma ferramenta de grande utilidade para a construção de tais indicadores, uma vez que seu objetivo central é o uso de métodos quantitativos para avaliar de forma objetiva a produção científica (ARAÚJO, 2006; OKUBO, 1997). 
Assim sendo, este trabalho se propôs a elaborar indicadores bibliométricos sobre a abordagem por competências no ensino de engenharia, a partir dos registros indexados à base de dados Web of Science. Foram analisados: número de publicações por ano; contribuição dos eventos científicos para o número de publicações; países, instituições e autores mais relevantes; diversidade de gênero entre os pesquisadores; publicação de maior impacto e tendências de pesquisa na área.

\section{METODOLOGIA}

Inicialmente foi realizada a seleção da base de dados a ser utilizada no trabalho, tendo sido escolhida a base de dados Web of Science devido a sua multidisciplinaridade, alcance internacional, fácil acesso, interface amigável, divisão do conteúdo em categorias, facilidade de exportação dos registros e disponibilidade de filtros para tratamento dos dados utilizando o software VantagePoint.

Uma vez selecionada a base, foi feito o estudo dos campos de pesquisa disponíveis, códigos associados a cada campo, categorias em que os assuntos estão divididos e funcionamento dos operadores booleanos, para então dar início ao processo de construção da expressão de busca.

A expressão de busca constitui um dos principais aspectos da construção dos indicadores bibliométricos, uma vez que é através dela que são definidos quais e quantos registros serão recuperados. É desejável, portanto, que a expressão de busca possua uma adequada precisão e revocação, de forma a recuperar o maior número de registros pertinentes ao tema buscado dentre a totalidade de registros existentes.

Desta forma, buscando abranger todas as expressões associadas tanto à Educação em Engenharia, quanto ao ensino por competências, foi empregada a metodologia "Building blocks", em que se separam as facetas do objeto de busca por tema, construindo grupos de palavras e expressões (quadro 1), que posteriormente serão conectados para compor a expressão de busca. Além disso, como a busca restringe-se ao ensino superior, foi necessário construir também um conjunto para excluir todas as expressões relativas aos demais níveis educacionais(SCHLOSSER et al., 2006). 
Quadro 1 - Grupos de palavras e expressões relevantes para a composição da expressão de busca.

\begin{tabular}{l|l|l|l}
\multicolumn{1}{c|}{$\begin{array}{c}\text { Designações de } \\
\text { competência }\end{array}$} & \multicolumn{1}{|c|}{$\begin{array}{c}\text { Competências } \\
\text { específicas }\end{array}$} & $\begin{array}{c}\text { Educação em } \\
\text { engenharia }\end{array}$ & \multicolumn{1}{|c}{$\begin{array}{c}\text { Outros níveis } \\
\text { educacionais }\end{array}$} \\
\hline Competencies & Communication & Engineering & Preschool \\
Employability skills & Creativity & STEM & Elergarten \\
Generic skills & Critical thinking & Education & Primary school \\
Key competencies & Entrepreneurship & Advisor & Elementary education \\
Non-technical skills & Ethics & Assessment & Primary education \\
Non-traditional skills & Innovation & Concepts & Middle school \\
Professional skills & Intercultural competence & Curricula & High school \\
Soft skills & Cross-cultural & Evaluation & K-12 \\
Transferable skills & competence & Exams & P-12 \\
Transversal competencies & Lifelong learning & Instruction & PK-12 \\
Twenty-first century skills & Problem solving & Learning & PreK-12 \\
$21^{\text {st }}$ century skills & Teamwork & Prepare & Pre-K-12 \\
& Leadership & Student & \\
& & Teaching & \\
& & Tests & \\
& & Training & \\
& & Tutor & \\
& & Accreditation & \\
& & Classroom & \\
& & Development & \\
& & Knowledge & \\
& & Rubric &
\end{tabular}

Fonte: Autoria própria, 2021.

Para a construção dos grupos, foi utilizada a metodologia "pearl growing", em que são buscadas em bases de dados todas as expressões relativas ao tema trabalhado. Para a composição do grupo relativo à Educação em Engenharia, fez-se uso da "Taxonomy of

Keywords for Engineering Education Research" (EER Taxonomy), enquanto os demais grupos foram construídos a partir da observação de termos recorrentes em publicações sobre competências e pesquisas na internet (FINELLI; BORREGO; RASOULIFAR, 2016; SCHLOSSER et al., 2006).

Utilizando os grupos contidos no quadro 1, foi realizada a construção da expressão de busca, seguindo as etapas presentes no quadro 2. 
Quadro 2 - Etapas utilizadas na construção da expressão de busca e seus respectivos resultados.

\begin{tabular}{|c|c|c|c|}
\hline Busca & Tópico & Expressão de busca & Resultados \\
\hline 1 & $\begin{array}{l}\text { Educação em } \\
\text { Engenharia }\end{array}$ & 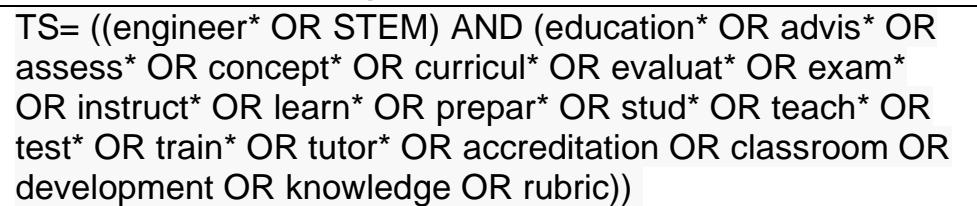 & 1.135 .850 \\
\hline 2 & $\begin{array}{l}\text { Removendo os } \\
\text { níveis } \\
\text { educacionais } \\
\text { indesejados }\end{array}$ & 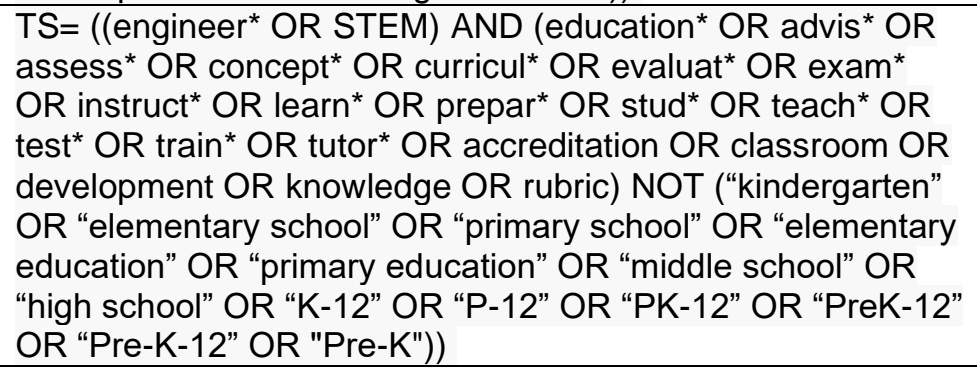 & 1.129 .498 \\
\hline 3 & $\begin{array}{l}\text { Designações para } \\
\text { competências }\end{array}$ & 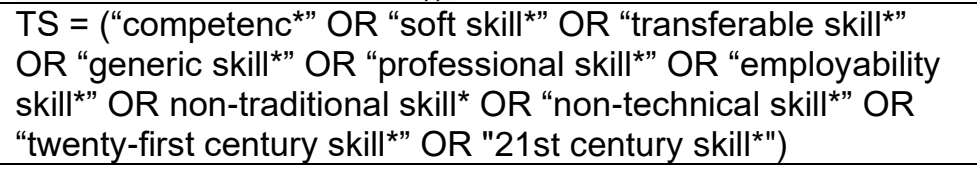 & 217.066 \\
\hline 4 & $\begin{array}{l}\text { Competências } \\
\text { específicas }\end{array}$ & $\begin{array}{l}\text { TS = ("communication" OR "creativity" OR "critical thinking" } \\
\text { OR "entrepreneurship" OR "ethics" OR "innovation" OR } \\
\text { "intercultural competence" OR "cross-cultural competence" } \\
\text { OR "lifelong learning" OR "problem solving" OR "teamwork" } \\
\text { OR "leadership") }\end{array}$ & 1.524 .725 \\
\hline 5 & $\begin{array}{l}\text { Combinando } \\
\text { competências e } \\
\text { educação e } \\
\text { engenharia }\end{array}$ & \#2 AND (\#3 OR \#4) & 57.266 \\
\hline 6 & $\begin{array}{l}\text { Incluindo as } \\
\text { categorias de } \\
\text { engenharia e } \\
\text { educação }\end{array}$ & $\begin{array}{l}\text { (\#2 AND (\#3 OR \#4)) AND WC = ("engineering" AND } \\
\text { "education") }\end{array}$ & 5.488 \\
\hline
\end{tabular}

Fonte: Autoria própria, 2021.

Em seguida, foi realizado um estudo da relevância dos resultados encontrados para avaliar a validade da expressão obtida, como pode ser visto no quadro 3. Para tal, determinou-se um período a ser analisado (2001-2020), de forma a restringir o número de registros para facilitar a análise, mas ainda garantir sua ocorrência em quantidade suficiente para determinar tanto o estado da arte, quanto a cronologia das publicações no tema. Em seguida, fez-se o ordenamento dos resultados por relevância (ferramenta disponível na própria base de dados) e foram examinados título e resumo dos 25 primeiros registros resultantes, para avaliar sua pertinência quanto ao tema pesquisado.

Quadro 3 - Estudo de relevância dos resultados obtidos pela utilização da expressão de busca.

\begin{tabular}{ccc}
\hline Expressão de busca & Resultados & Relevantes \\
\hline (\#2 AND (\#3 OR \#4)) AND WC = ("engineering" AND "education") & 5.165 & $25 / 25$ \\
\hline
\end{tabular}

Fonte: Autoria própria, 2021.

Foi possível constatar a pertinência de todos os registros da amostra analisada, comprovando a adequação da expressão de busca utilizada. Por fim, os resultados foram exportados para o software VantagePoint v5.0, no qual foi realizado o tratamento dos dados 
para padronização e análise, tendo sido utilizado o software de planilhas eletrônicas Microsoft Excel para a construção dos gráficos apresentados abaixo.

\section{RESULTADOS E DISCUSSÃO}

A partir dos dados recuperados na base Web of Science sobre o número anual de publicações relativas à utilização do ensino por competências na Educação em Engenharia, temos a figura 1.

Figura 1 - Número de publicações por ano, nos últimos 20 anos (2001-2020).

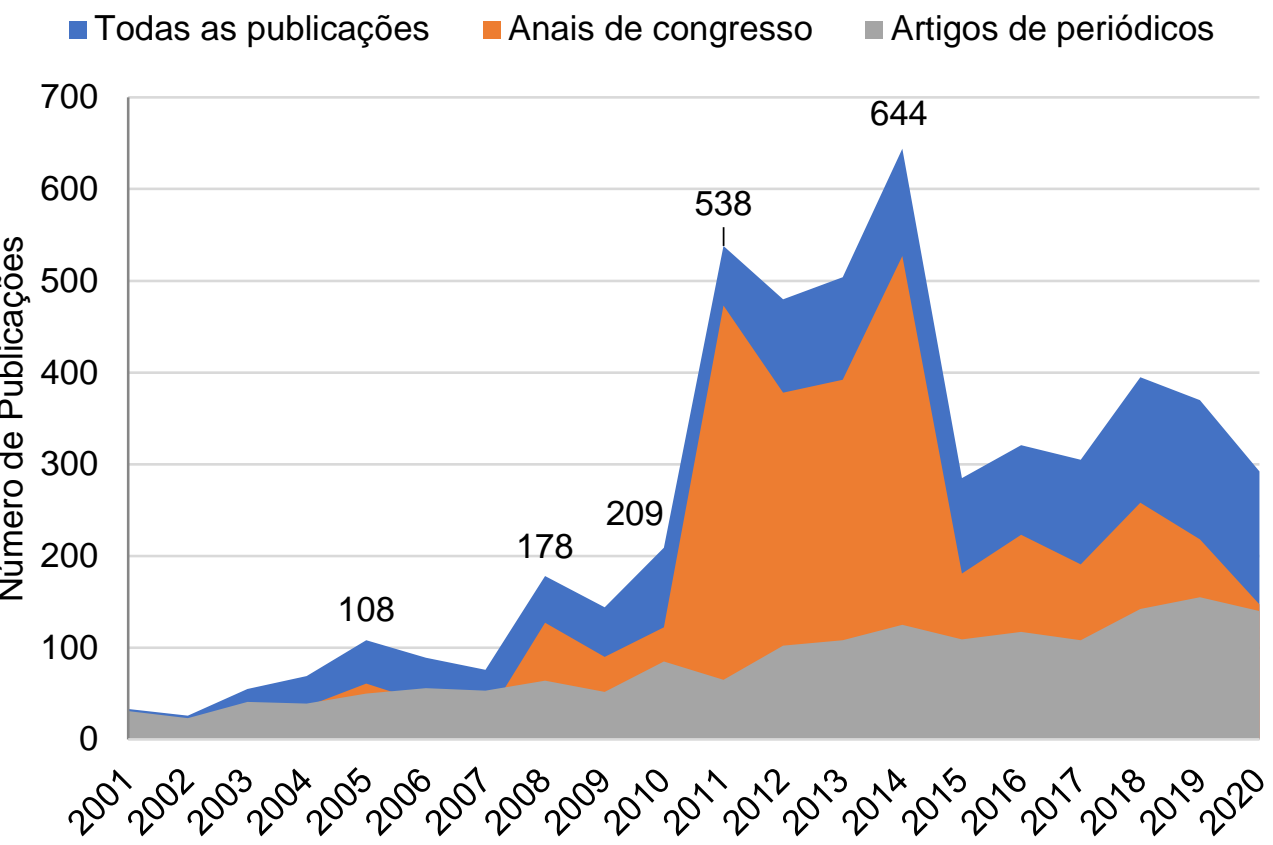

Fonte: Os autores, 2021.

O número total de publicações por ano, representado no gráfico pela cor azul, permite observar uma tendência de crescimento das publicações, mesmo que pouco expressiva, do ano de 2001 ao ano de 2010, apresentando picos de crescimento nos anos de 2005, 2008 e 2010. Já na segunda metade do gráfico (2011-2020) notam-se resultados mais expressivos, com um aumento de mais de duas vezes no ano de 2011 , se comparado ao maior pico presente no primeiro trecho analisado (2010), e um aumento de mais de três vezes no ano de 2014.

Além disso, enquanto o número de publicações de artigos em periódicos, representado em cinza na figura 1, possui um crescimento discreto em todo o período analisado, variando de 23 publicações, em 2002, até 155 publicações, em 2019; a fração de registros dos anais de congressos, representada pela cor laranja, permite de imediato verificar as semelhanças entre seus picos e os do número total de publicações. Desta forma, podemos destacar a importância destes eventos ao tema de pesquisa, como geradores de conteúdo para a área e facilitadores na troca de informações entre pesquisadores de diferentes instituições e países, bem como ressaltar a importância do ensino por competências, uma vez que este vem sendo abordado em diversos eventos, como mostra a figura 2. 
Figura 2 - Número de publicações por ano (2001-2020) associados a eventos de Educação em Engenharia.

$\llbracket$ Anais de congresso $\square$ ASEE Annual Conference $\backsim$ Frontiers in Education (FIE)

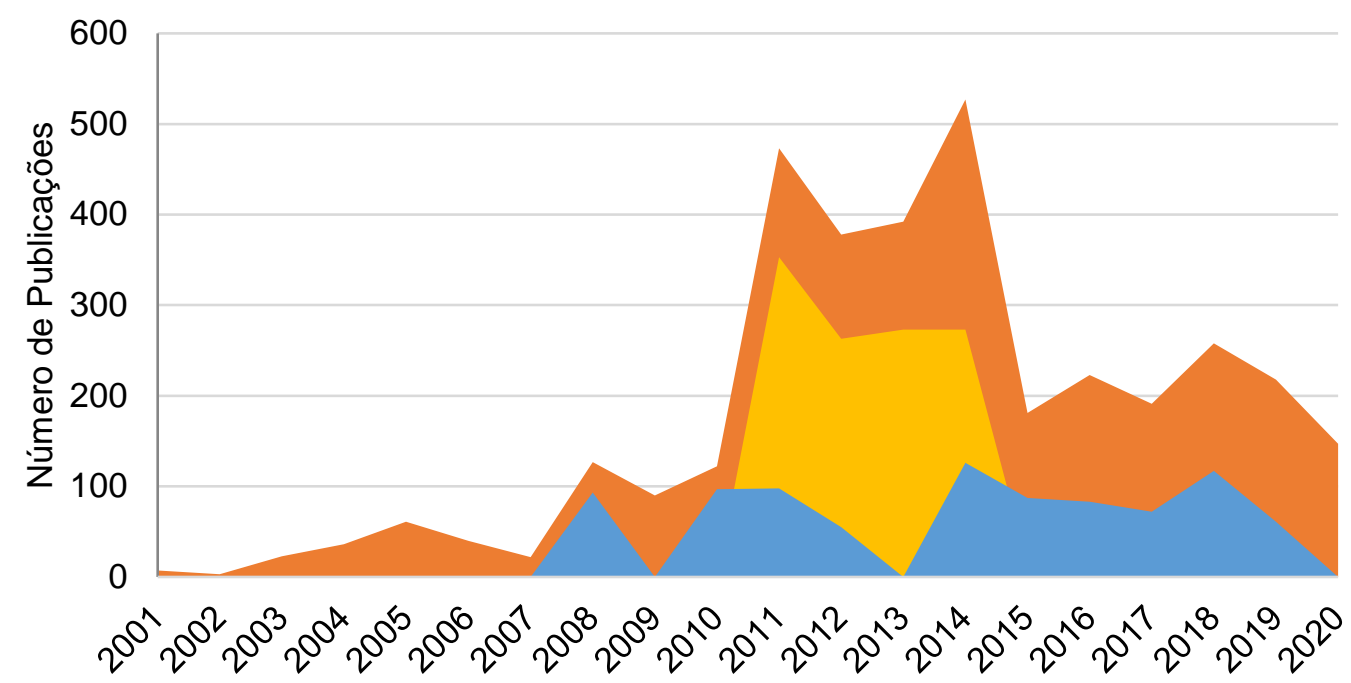

Fonte: Os autores, 2021.

Uma análise mais detalhada dos resultados retornados, permitiu associar o aumento no número publicações à indexação dos anais de dois grandes eventos. A "ASEE Annual Conference" é um importante e reconhecido evento na área da Educação em Engenharia, que aborda os assuntos relevantes ligados ao tema. Já o "Frontiers in Education" (FIE), organizada pelo Institute of Electrical and Electronics Engineers (IEEE), que é maior organização profissional técnica do mundo; oferece um fórum acadêmico internacional para a discussão de abordagens educacionais baseadas em pesquisas nas mais diferentes áreas, entre elas, o STEM (Science, technology, engineering e mathematics). Como dito anteriormente, a presença de um congresso diretamente ligado à Educação em Engenharia e um relacionado à educação no âmbito geral como maiores participantes nas publicações relacionados ao ensino por competências, reforça mais uma vez a relevância do tema, uma vez que este vem sendo abordado em variados tipos de eventos.

Dando continuidade à análise dos dados recuperados, estes foram agrupados quanto ao país de origem, como mostra a figura 3. 
Figura 3 - Número de publicações por país, para o período de 2001 a 2020.

Número de Publicações

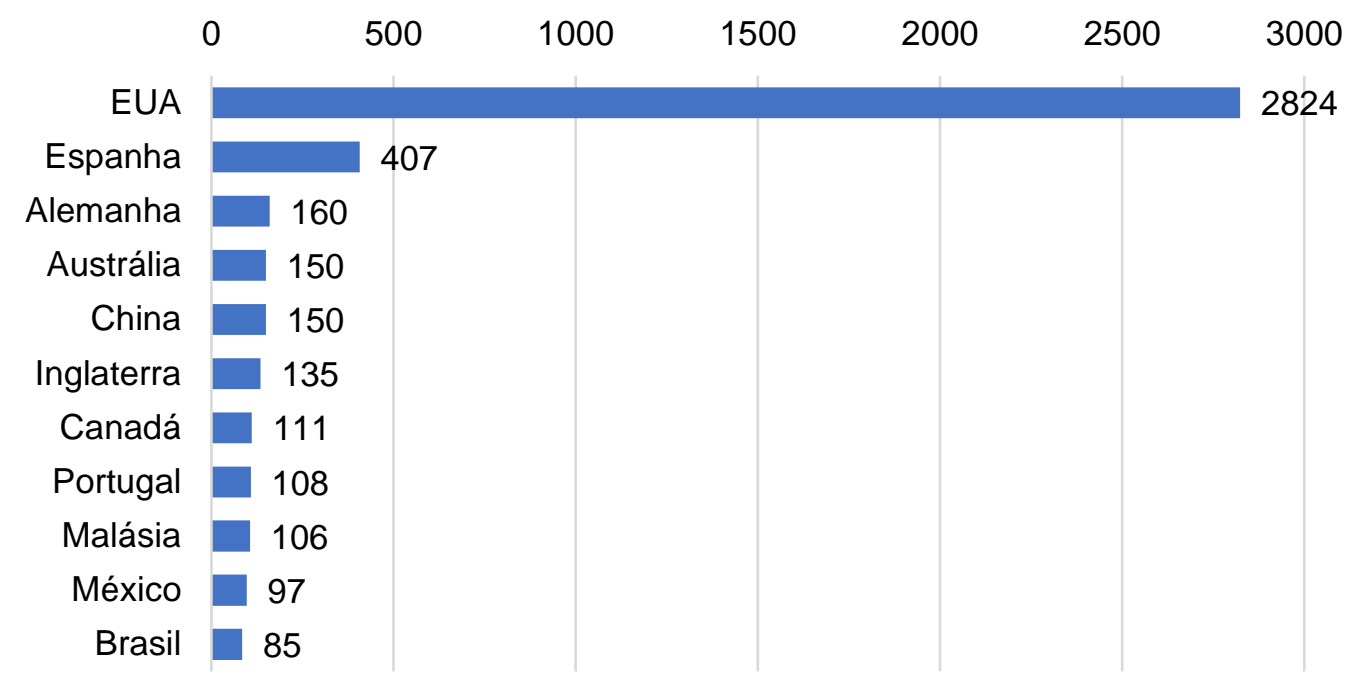

Fonte: Os autores, 2021.

Mostra-se evidente a dominância dos EUA no que se refere às pesquisas relacionadas ao ensino por competências na Educação em Engenharia, somando 2.824 trabalhos indexados à base de dados, o que representa $49,2 \%$ do total de publicações analisadas e quase sete vezes mais que número de publicações da Espanha, segundo colocado, com 407 registros. Os demais países apresentam uma quantidade inferior à 200 publicações na área, para o período.

O Brasil, por exemplo, aparece na $11^{\circ}$ posição, dentre os 106 países analisados, com um total de 85 publicações. Embora não seja uma posição muito baixa, seu número de publicações é o equivalente a apenas $1,5 \%$ do total de publicações analisadas, o que não constitui uma contribuição expressiva para o número total de publicações, representando a necessidade de um maior investimento neste tema de pesquisa, dada a sua importância, discutida anteriormente. Felizmente, a implantação das DCNs e vigência do PMG-EUA, têm favorecido a ocorrência de discussões sobre o ensino por competências, o que pode promover um aumento no número de publicações nessa área nos próximos anos.

Quanto ao número de publicações por instituição de ensino, como pode ser visto na figura 4, a dominância dos EUA se mantém. 
Figura 4 - Número de publicações por instituição de ensino, para o período de 2001 a 2020.

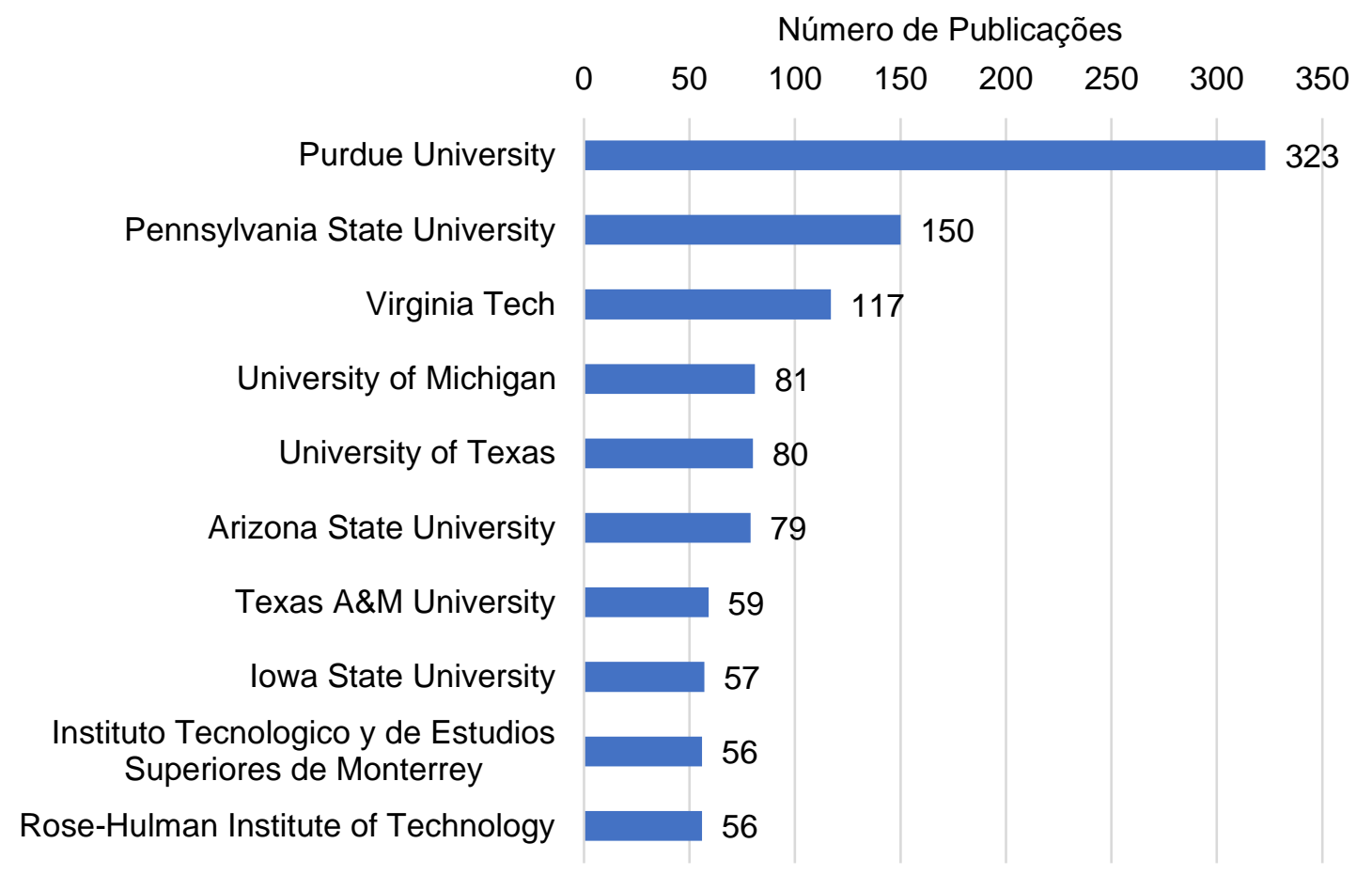

Fonte: Os autores, 2021.

Dentre as dez instituições com maior número de publicações, nove são localizadas nos EUA e uma no México, sendo a Purdue University responsável pelo maior número de publicações (323), seguido pela Pennsylvania State University (150) e Virginia Tech (117).

Com intuito de se entender tanto a razão do elevado número de publicações por tais instituições, quando da significativa dominância dos EUA neste campo de pesquisa, investigações adicionais mostraram a ocorrência, tanto de cursos de pós-graduação específicos em Educação em Engenharia em diversas instituições estadunidenses, quando a existência departamentos dedicados exclusivamente à Educação em Engenharia em algumas delas, como: Purdue University, Virginia Tech, University of Texas e Arizona State University.

A figura 5 apresenta um panorama geral sobre os autores com maior número de publicações na área. 
Figura 5 - Número de publicações por instituição de ensino, para o período de 2001 a 2020.

Número de Publicações

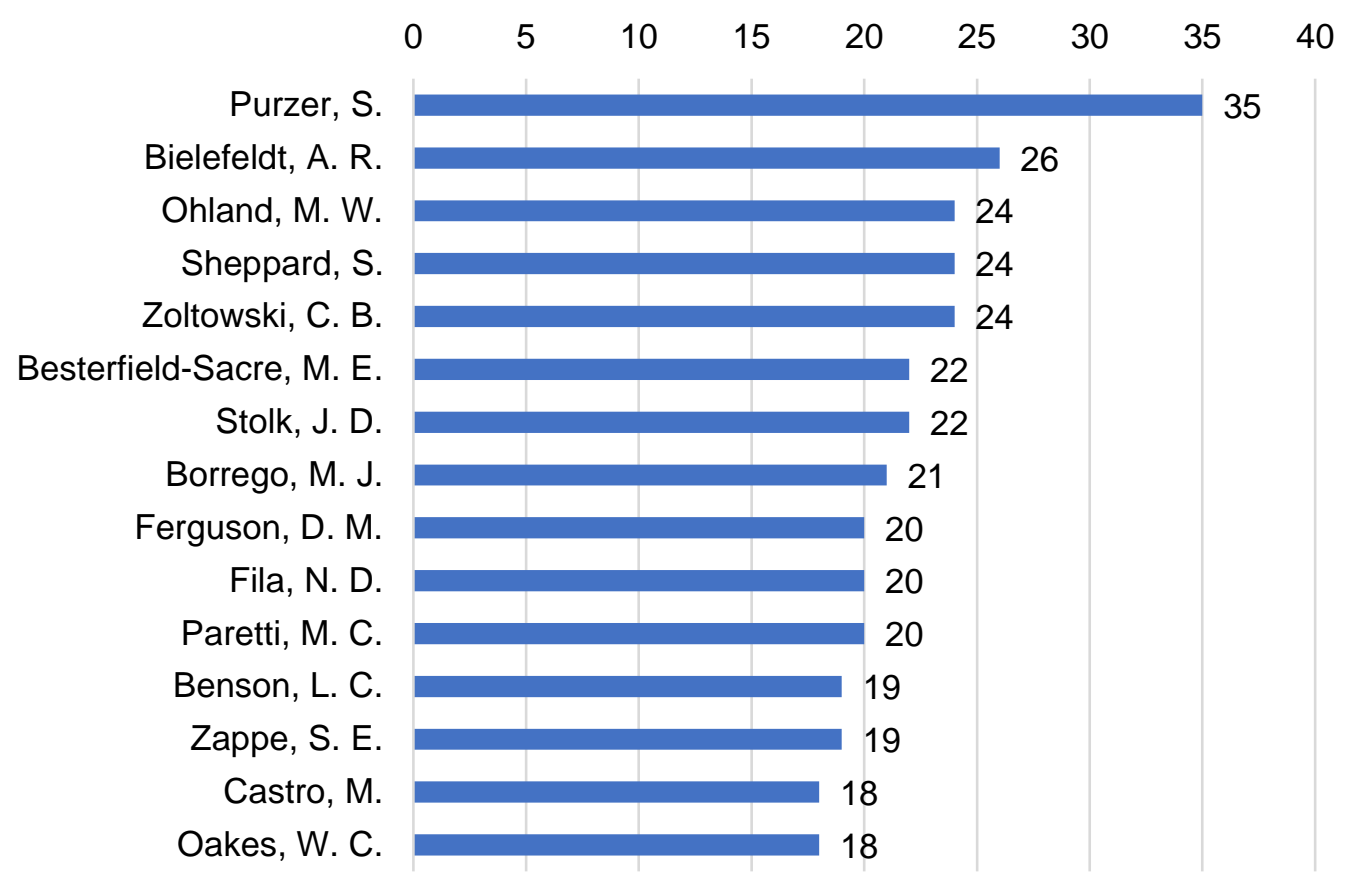

Fonte: Os autores, 2021.

Dentre os 15 pesquisadores presentes na lista, 14 atuam nos EUA, mantendo a coerência com os comportamentos observados nas figuras 3 e 4. Além disso, o único pesquisador vinculado à Espanha, dentre os 15 autores mencionados, também acompanha a tendência vista na figura 3, uma vez que o país é o segundo em número de publicações no período.

A pesquisadora Senay Purzer, professora de Educação em Engenharia na Purdue University, apresenta o maior número de publicações (35 no total). O segundo nome listado, com 26 publicações, é Angela R. Bielefeldt, professora de Engenharia Civil e Ambiental e pesquisadora em Educação em Engenharia, na University of Colorado. Em seguida, com 24 publicações, temos Matthew W. Ohland, da Purdue University; Sheri D. Sheppard, da Stanford University e Carla B. Zoltowski, também da Purdue University. Inclusive, dentre os 15 autores com maior número de publicações, 5 são afiliados à Purdue University, não havendo repetição entre as demais instituições de afiliação, o que reforça os resultados observados na figura 4.

Outro dado de interesse a ser discutido é a significativa representatividade feminina na área de Educação em Engenharia, neste recorte do Ensino por Competências. Embora a Engenharia seja muitas vezes reconhecida como uma área predominantemente masculina, entre os 15 pesquisadores com maior número de publicações, 60\% são mulheres, sendo estas responsáveis, inclusive, pelos dois maiores números de publicações.

E interessante mencionar também, que a pesquisadora Mary E. Besterfield-Sacre, que ocupa a 6" posição no número de publicações, é uma das autoras do artigo "The $A B E T$ "Professional skills" - Can they be taught? Can they be assessed?", publicado pelo Journal of Engineering Education, em 2005, e que apresenta o maior número de citações no período analisado (476 citações), constituindo uma importante referência na área. Além disso, a pesquisadora foi selecionada pelo PMG-EUA, como a especialista encarregada das 
oficinas de ensino por competências, voltadas aos professores das IES brasileiras que participam do programa.

Quanto à identificação dos direcionamentos de pesquisa na área, na figura 6, temos as palavras-chave de maior ocorrência nos registros analisados no período.

Figura 6 - Palavras-chave de maior ocorrência.

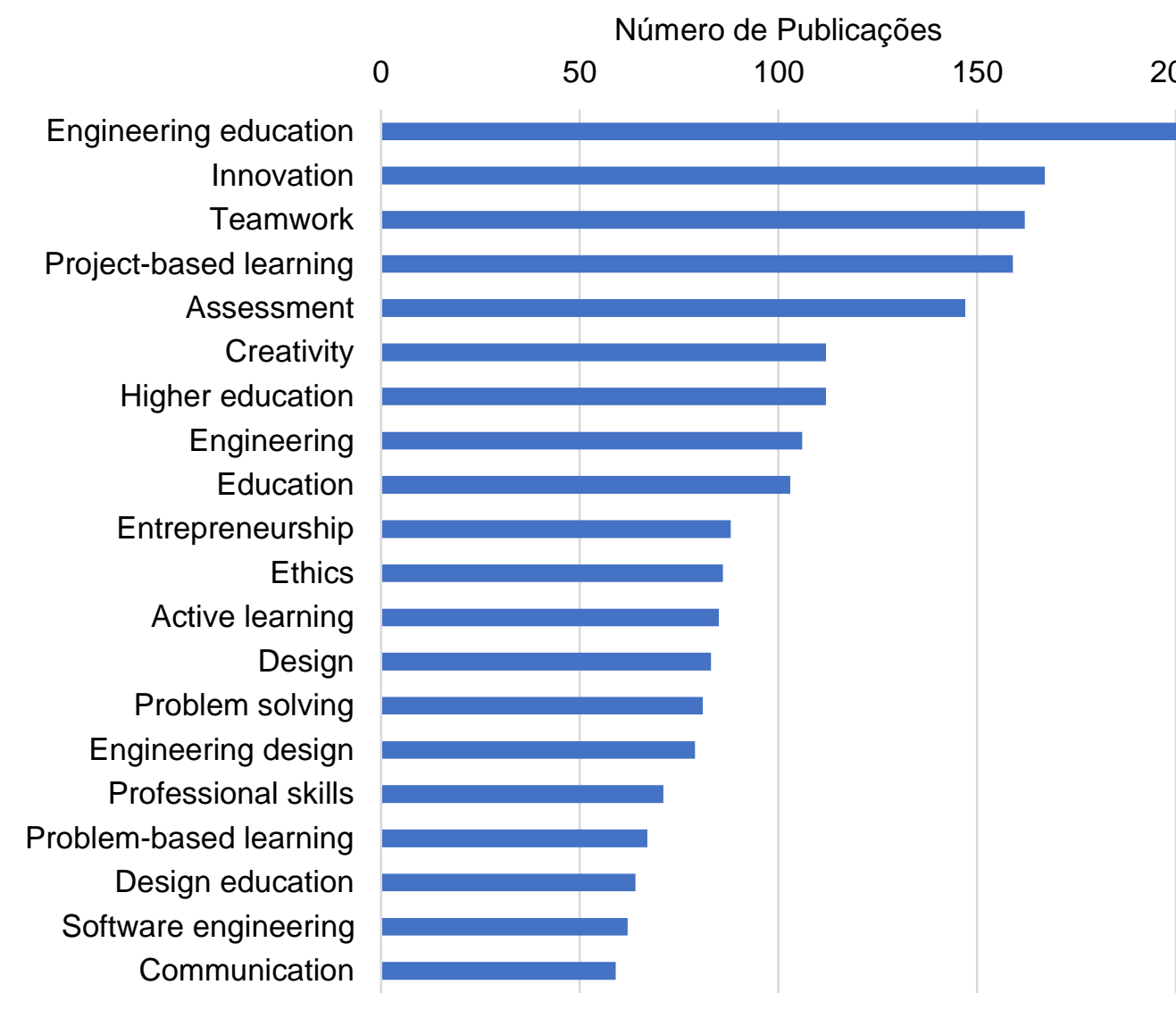

Fonte: Os autores, 2021.

Embora a avaliação da recorrência de palavras-chave retorne resultados genéricos como engineering e education, é possível observar também a presença de termos relacionados às competências (teamwork, creativity, entrepreneurship, entre outros), como já era de se esperar, devido ao escopo da pesquisa. Pode-se notar ainda, a ocorrência de palavras-chave relacionadas ao uso de metodologias ativas de ensino-aprendizagem, como project-based learning, active learning e problem-based learning, indicando uma proximidade entre o ensino por competências e o uso de metodologias ativas, uma vez que estas constituem ferramentas modernas de ensino, que tem o aluno como agente fundamental, favorecendo a construção de competências.

\section{CONCLUSÃO}

O estudo dos indicadores bibliométricos sobre o ensino por competências na Educação em Engenharia, permitiu traçar o panorama desta área de pesquisa, contextualizar a produção científica da área em âmbito mundial, reconhecer os principais autores e instituições, verificar a diversidade de gênero entre os pesquisadores, delinear tendências de pesquisa no campo e identificar aspectos a serem abordados em trabalhos futuros. 
Foi possível observar, primeiramente, o crescimento do número de publicações ao longo do tempo, no período analisado (2001 a 2020), com destaque para as publicações advindas de grandes eventos de Educação em Engenharia.

Os EUA representam o maior expoente neste campo de pesquisa, sendo responsável por quase $50 \%$ de todas as publicações na área, tendência que se mantém quanto às instituições e pesquisadores de destaque, de forma que, dentre as dez instituições com maior contribuição para o total de publicações, apenas uma não está localizado nos EUA e dentre os 15 pesquisadores mais ativos na área, apenas um não atua no país. O Brasil é responsável por apenas uma pequena parcela das publicações analisadas, demonstrando a necessidade de desenvolvimento da temática no país.

A Purdue University, apresenta o maior número de publicações, seguida pela Pennsylvania State University e a Virginia Tech, sendo que entre as dez instituições com mais publicações, apenas uma não se localiza nos EUA.

Quanto ao número de publicações por autor, destacam-se as pesquisadoras Senay Purzer e Angela R. Bielefeldt, com os maiores número de publicações. Entre os 15 autores apresentados, 9 são mulheres, indicando a diversidade de gênero entre os pesquisadores e a predominância feminina neste campo de pesquisa.

No que tange as temáticas de pesquisa envolvendo o ensino por competências na Educação em Engenharia, a análise da recorrência de palavras-chave permitiu identificar a tendência em pesquisas sobre o uso de metodologias ativas de ensino-aprendizagem. Tal tendência abre portas para a proposição de trabalhos futuros com o intuito de identificar quais as metodologias ativas mais utilizadas e as melhores formas de avaliar sua efetividade.

\section{Agradecimentos}

Ao CNPq - Conselho Nacional de Desenvolvimento Científico e Tecnológico pelo apoio financeiro para realização desse trabalho com bolsa de estudos, processo 143364/2019-7.

À CAPES - Coordenação de Aperfeiçoamento de Pessoal de Nível Superior pelo apoio financeiro para realização desse trabalho com bolsa de estudos, processo $\mathrm{n}^{\circ}$ 88887.494624/2020-00.

O presente trabalho foi realizado com apoio da Coordenação de Aperfeiçoamento de Pessoal de Nível Superior - Brasil (CAPES) - Código de Financiamento 001.

\section{REFERÊNCIAS}

ARAÚJO, C. A. Bibliometria. Em Questão, Porto Alegre, v. 12, n. 1, p. 11-32, 2006. Disponível em: <https://seer.ufrgs.br/EmQuestao/article/view/16/5>. Acesso em: 17 jan. 2021.

BRASIL. Diretrizes Curriculares Nacionais do Curso de Graduação em Engenharia. . 23 jan. 2019, 1.

CAPES; COMISSÃO FULBRIGHT. Programa Brasil-Estados Unidos de modernização da educação superior na graduação (PMG-EUA). . 11 jun. 2018.

CNI; SESI; SENAI; IEL; CNE; ABENGE; CONFEA. Documento de apoio à implantação das DCNs do curso de graduação em engenharia. Brasília: CNI, 2020. Disponível em: https://static.portaldaindustria.com.br/media/filer_public/95/2b/952b2b44-3671-4556-bfa8- 
d0f446185a29/documento_de_apoio_implantacao_dcns_curso_graduacao_engenharia.pd f

FINELLI, C. J.; BORREGO, M.; RASOULIFAR, G. Development of a taxonomy of keywords for engineering education research. European Journal of Engineering Education, [s. I.], v. 41, n. 3, p. 231-251, 2016. Disponível em:

<https://www.tandfonline.com/doi/full/10.1080/03043797.2016.1153045>. Acesso em: 17 abr. 2020.

OKUBO, Y. Bibliometric indicators and Analysis of research systems: Methods and

Examples: OECD science, technology and industry working papers. Paris:

ORGANISATION FOR ECONOMIC CO-OPERATION AND DEVELOPMENT, 1997.

Disponível em: https://www.oecd-ilibrary.org/science-and-technology/bibliometric-

indicators-and-analysis-of-research-systems_208277770603. Acesso em: 16 jun. 2020.

SCHLOSSER, R. W.; WENDT, O.; BHAVNANI, S.; NAIL-CHIWETALU, B. Use of information-seeking strategies for developing systematic reviews and engaging in evidence-based practice: the application of traditional and comprehensive Pearl Growing. A review. International Journal of Language \& Communication Disorders, [s. I.], v. 41, n. 5, p. 567-582, 2006. Disponível em: http://doi.wiley.com/10.1080/13682820600742190. Acesso em: 9 maio. 2021.

\title{
BIBLIOMETRIC INDICATORS OF THE COMPETENCIES APPROACH IN ENGINEERING EDUCATION
}

\begin{abstract}
In order to meet the demands of the job market and modernize undergraduate engineering courses, in 2019, the new Diretrizes Curriculares Nacionais do Curso de Graduação were published, based on a new teaching concept focused on building competencies. In addition, the Programa Brasil-Estados Unidos de Modernização da Educação Superior na Graduação is in effect, which aims, among other objectives, to foster the construction of competencies such as creativity, innovation and entrepreneurship. Thus, there is an urgent need for a greater understanding of the application of competency-based education in engineering education. In order to clarify this topic and determine its scientific impact, the proposal of this paper is to develop bibliometric indicators, based on the records available in the Web of Science database, from 2001 to 2020. The number of publications per year was analyzed, showing an increasing number of publications over time. In addition, regarding productivity by country, institution and author, the USA was predominant in this field of research, being the country responsible for the vast majority of records in the analyzed period. There is a significant female representation in the area. Futhermore, could be observe the detection of research trends that combine the competencies approach with the use of active methodologies, being the theme for the next works, a more detailed investigation about which active methodologies are most used and which are the best methods to assess its effectiveness.
\end{abstract}

Keywords: Competencies. Engineering education. Bibliometrics. Bibliometric indicators. 